\title{
CANALES DE DISTRIBUCIÓN EN LAS VENTAS DE LIMÓN DE LOS PRODUCTORES DE LA PARROQUIA AYACUCHO, CANTÓN SANTA ANA, PROVINCIA DE MANABÍ
}

\author{
DISTRIBUTION CHANNELS IN THE LEMON SALES OF THE PRODUCERS OF \\ THE AYACUCHO PARISH, SANTA ANA CANTON, MANABÍ PROVINCE
}

\author{
Ruth Sumba-Bustamante ${ }^{1}$, María Vinueza-Ramirez ${ }^{2}$, Thalia Pibaque-Molina ${ }^{3}$ \\ 1,2,3 - Universidad Estatal del Sur de Manabí, Manabí, Ecuador
}

1. Email: yadira.sumba@unesum.edu.ec ORCID: https://orcid.org/0000-0003-2620-1129

2. Email: lissethvinueza@hotmail.com ORCID: https://orcid.org/0000-0003-4665-3114

3. Email: pibaque-molina1864@unesum.edu.ec ORCID: https://orcid.org/0000-0003-1710-5207

Recibido: 11/05/2021 Aceptado: 23/07/2021

Para Citar: Sumba-Bustamante, R., Vinueza-Ramirez, M., \& Pibaque-Molina, T. (2021). Canales de distribución en las ventas de limón de los productores de la parroquia Ayacucho, cantón Santa Ana, provincia de Manabí. Revista Publicando, 8(31), $240-257$. https://doi.org/10.51528/rp.vol8.id2247

\begin{abstract}
Resumen:
Un canal de distribución consiste en el conjunto de personas y empresas comprendidas en la transferencia de derechos de un producto, incluye siempre al productor y al cliente final del producto en su forma presente, así como a cualquier intermediario, como los detallistas y mayoristas, su importancia radica en el beneficio que reciben los consumidores en cuanto al ahorro de tiempo para satisfacer necesidades con la obtención del producto, sin embargo se ha observado que en la parroquia Ayacucho, la comercialización del limón es realizada utilizando varios eslabones en el canal de distribución obstaculizando la obtención de mayores ingresos a los productores. En razón a lo cual en el presente estudio se planteó determinar los canales de distribución que aportan al incremento de las ventas de limón en la parroquia, para lo cual se utilizó la investigación exploratoria, de campo y documental, con las técnicas de entrevista a la Presidenta del GAD Parroquia y encuesta a 49 productores, estableciendo el canal de distribución que utilizan, y recomendando la implementación de alianzas estratégicas que contribuyan a fomentar la exportación de su producto para la dinamización de la actividad comercial.
\end{abstract}

Palabras clave: canal de distribución, limón, productores, consumidor, ventas.

\begin{abstract}
:
A distribution channel consists of a set of people and companies involved in the transfer of rights of a product, it always includes the producer and the final customer of the product in its present form, as well as any intermediary, such as retailers and wholesalers, its importance lies in the benefit that consumers receive in terms of saving time to meet needs with obtaining the product, however it has been observed that in the Ayacucho parish, the commercialization of lemon is done using several links in the distribution channel hindering the obtaining of higher income to producers. For this reason, in this study it was proposed to determine the distribution channels that contribute to the increase of lemon sales in the parish, for which exploratory, field and documentary research was used, with the techniques of interviewing the President of the Parish GAD and a survey of 49 producers, establishing the distribution channel they use, and recommending the implementation of strategic alliances that contribute to promote the export of their product for the revitalization of commercial activity.
\end{abstract}

Keywords: distribution channel, lemon, producers, consumer, sales. 


\section{INTRODUCCIÓN}

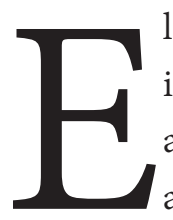

sector agropecuario tiene una importancia vital para todos los países, ayuda a compensar la necesidad de alimentación de las poblaciones, entre estos productos se destacan los cítricos como el limón. Se considera un fruto que se encuentra disponible todo el año, pero su abundancia se da en los meses de septiembre a enero. Es originario de Asia de donde también fue llevado al sur de Francia, Grecia y California (Estados Unidos) y se presume que fue introducido a Ecuador en la época de la conquista española. Es jugoso, tiene mucha vitamina $\mathrm{C}$, ácido cítrico, sustancias de acción astringente y potasio, su mineral más abundante (Arreaga Gómez, 2017).

Es producido por varios países, entre los cuales para el año 2019 se consideró La India como el mayor productor de limón con 3.482 toneladas métricas, seguido por México y China, con la segunda y tercera ubicación respectivamente (Statista, 2021). Sin embargo, los mayores exportadores de limones del mundo en ese mismo año fueron España y México, con envíos por 828.6 millones y 523.1 millones de dólares respectivamente (Opportimes, 2020).

Se han realizado estudios en algunos países de América y en Ecuador, relacionados con el problema planteado, como es la investigación titulada La producción y la comercialización del limón persa: una alternativa para el desarrollo de la región bajo mixe, Oaxaca, realizada por Espejo Martínez y Ramos Soto (2017) que tuvo como objetivo describir las condiciones socioeconómicas de los productores de limón persa de la región Bajo Mixe, a fin de identificar las fortalezas y los problemas a los que se enfrentan los productores al producir y comercializar su producto $y$, al mismo tiempo, distinguir qué oportunidades tienen para destacar como una zona citrícola, implementando la metodología cuantitativa (encuestas) obteniendo como resultado que la producción de este fruto ha permitido solventar sus necesidades básicas de alimento, vestido y tener una vivienda con los servicios básicos, asimismo se identificó que predominan productores pequeños en la zona de estudio, como principales debilidades sobresalen la falta de organización de los productores y las limitaciones en los procesos de comercialización.

Así mismo De la Riva Del Valle (2018) en su investigación titulada Estudio de mercado de limón persa (Citrus latifolia) en la ciudad de Guatemala que tuvo como objetivo documentar el comportamiento del mercado de limón persa en la ciudad de Guatemala, con el propósito de informar a productores, intermediarios y consumidores, sobre la tendencia de la oferta, demanda y precio en la época seca y lluviosa del país, obtuvo como resultado que los precios varían entre la época seca y época lluviosa del país por la ausencia de agua en las parcelas de cultivo puesto que en época seca es escasa porque una gran parte del área del cultivo no cuenta con sistemas de riego eficientes por lo cual el árbol cuenta con poca producción durante estos meses del año y esta escases del limón hace que el precio aumente y en relación con la época lluviosa la producción es abundante por lo cual el precio disminuye.

Por otra parte Kennedy (2014) en la investigación titulada Estudio de mercado y prefactibilidad del cultivo de limón TAHITI (Citrus aurantifolia) en la Provincia de Santa Elena 
que tuvo como objetivo el estudio de mercado y pre-factibilidad del cultivo de Limón Tahití en las Provincias del Guayas y Santa Elena, obtuvo como resultado que en el Ecuador destina la mayor parte de su producción de limón al mercado de fruta fresca tanto para el consumo interno como para la exportación, lo que determina que en el país no existe una verdadera industria procesadora de limón y que el principal mercado de destino es a los Estados Unidos.

Se debe agregar que Santistevan Mendez (2016) en la investigación titulada La sustentabilidad del cultivo del limón (Citrus aurantifolia (Christm) S, ) en la provincia Santa Elena, Ecuador que tuvo como objetivo conocer el comportamiento del cultivo de limón (Citrus aurantifolia Swingle) en dos localidades de la Provincia de Santa Elena, Ecuador, implementando la metodología del "análisis multicriterio" que permite calcular el Indicador Económico (IE), Indicador Ecológico (IE) y el Indicador Social Cultural (ISC) de cada finca, con cuyos datos se estima el Indicador de Sustentabilidad General (IS Gen) de la misma obteniendo como resultado que las fincas limoneras son muy complejas y que las familias tienen una alta dependencia de este cultivo.

El limón puede ser cultivado en terrenos de textura arcillosa con temperaturas de hasta 40 grados, en Ecuador de acuerdo con Pino et al. (2009) se producen dos variedades: el limón "sutil" y el limón "tahiti", el primero se cultiva principalmente para abastecer la demanda local, en cambio el segundo se destina a la exportación casi en su totalidad, únicamente se introduce en el mercado local, cuando hay escasez del limón sutil.
Ecuador debido a su ubicación territorial se encuentra en una posición favorable para la producción de limón, todas las regiones del país cuentan con ambientes climáticos y ambientales adecuados para el cultivo. Según la Encuesta de Superior y Producción Agropecuaria Continua (ESPAC) en el año 2018 fueron 5.726 hectáreas plantadas, 4.619 hectáreas cosechadas, 24.144 de producción en toneladas métricas que generó unas ventas de 23.143 en toneladas métricas, las Provincias que producen más son Guayas y Manabí (Instituto Nacional de Estadísticas y Censos. Encuesta de Superficie y Producción Agropecuaria Continua, 2019).

Según la FAO, Ecuador en el 2010 y 2011, logró producir 115.2 y 100.6 mil toneladas métricas de cítrico. Datos del Banco Central del Ecuador, señalan que, entre limón Tahití, sutil, y lima se exporto 6.622 toneladas métricas y a diferentes partes del mundo (Dirección de Inteligencia Comercial e Inversiones, 2013).

La producción llega al mercado, debido a que Ecuador no cuenta con procedimientos de transformación de este crítico. Se exporta para su consumo e industrialización en mercados como los Estados Unidos, Francia, entre otros (Vásquez \& Vásquez, 2014).

La producción de limón en Ecuador se ve afectada por el clima frío, las lloviznas, debido a que cuando hay garuas disminuye la floración y por ende la producción es un tema estacional puesto que en el verano se incrementa, las plagas en ocasiones dañan la cosecha perjudicando la calidad del producto (Ecuavisa, 2017).

La mayor producción de limón en Manabí se encuentra en el valle del rio de Portoviejo con 1200 
hectáreas, por lo general este producto se encuentra en el mercado todo el año, pero abunda más de septiembre a febrero, las cosechas de limón sutil y criollo se venden un $65 \%$ a las cadenas de supermercados (El Comercio, 2011).

La producción de limón en la parroquia Ayacucho del Cantón Santa Ana es realizada por pequeños y medianos productores que manejan un canal de distribución inadecuado, venden a través de intermediarios, debido a: la carencia de conocimientos de comercialización, poca asociatividad por parte de los productores o la poca gestión para vender a mejores precios y cantidades.

Los canales de comercialización afectan las condiciones de ingresos de los productores, al existir algunos intermediarios dentro del proceso de comercialización del producto los ingresos que perciben por sus ventas son mínimos lo que ocasiona baja rentabilidad de su producción y poca actividad comercial dentro del mercado, situación observada que permitió formular el siguiente problema de investigación ¿Cuál es el canal de distribución que aporta al incremento de las ventas de limón en la parroquia Ayacucho?.

Para el desarrollo de este estudio se utilizó la investigación bibliográfica, exploratoria y de campo, para la toma de información se recurrió a la encuesta y a la entrevista, que permitieron obtener los resultados alcanzados.

\section{CANALES DE DISTRIBUCIÓN}

Según Lamb et al. (2011) un canal de marketing (también llamado canal de distribución) es una estructura de negocios de organizaciones interdependientes que participan en el proceso de tener disponible un producto o servicio para el uso de los consumidores finales, idea que coincide con lo expresado por Armstrong y Kotler (2013) quienes consideran que es un conjunto de instituciones interdependientes que ayudan a que un producto o servicio se encuentre disponible para su uso o consumo por el comprador o el usuario corporativo.

Frecuentemente, los canales de distribución parecen bastante estables, sin embargo, cada canal de distribución va cambiando. Nuevos intermediarios como las organizaciones que ayudan a conectar al productor con el consumidor surgen en algunos canales para prestar nuevos servicios. En otras fases los intermediarios van desapareciendo en lo posible los productores se acercan a los minoristas o incluso directamente a los consumidores. Aun en otros canales un tipo de minoristas o distribuidor reemplaza a otro tipo. A pesar de que los cambios son lentos, los giros de tendencias en distribución son poderosos. La razón principal para simplificar la cadena de distribución es que cada intermediario impone su precio, lo cual les cuesta dinero al productor y al consumidor (Figueroa, 2015).

Un canal de comercialización es de mucha importancia en el mercado, debido a que: permite que se conozca información respecto a los compradores, productores y otros actores que intervienen en el intercambio; desarrollan y difunden comunicaciones respecto a la oferta de un producto para satisfacer las necesidades del consumidor.

Existen algunos tipos de canales de distribución, entre los cuales se encuentran los siguientes según Diez de Castro y Navarro García (1994). 
Canal de bienes de consumo: Este tipo de canal es empleado para el traslado de productos físicos desde el fabricante a los consumidores finales donde se puede emplear diversas alternativas para la comercialización de los bienes.

Canal industrial o de bienes industriales: Este canal se produce en el traslado de productos físicos, pero con la esencia de que sean asociados al proceso productivo de las empresas o al crecimiento de las labores industriales, es decir no se procede en el mercado de consumo sino en el organizacional.

Canal de servicios: La esencia de esta transacción no es un producto material, sino de un servicio, en este asunto los destinarios del servicio pueden ser las clientelas finales o también los industriales suelen ser habituales el empleo de canales directos, sin embargo, la importancia de los intermediarios se va resaltando cada vez más.

Cada tipo de canal de distribución presenta niveles, los mismos se detallan a continuación (Velazquez Velazquez, 2012).

\section{Niveles de canales de bienes de consumo}

Fabricante - consumidor: Es también llamado canal directo, debido que no cuenta con niveles de intermediarios y los productores venden directamente a los compradores.

Productores - minoristas o detallista consumidores: Se designa como canal dos, debido a que tiene un nivel de intermediarios, a saber: los minoristas o detallistas.

Productores - mayoristas - minoristas o detallistas - consumidores: Este tipo de canal tiene dos niveles de intermediarios y se le denomina como canal tres, puesto que se utiliza con reiteración en los pequeños comerciantes de alimentos y de medicinas, o en aquellos productores que no tienen la capacidad de hacer llegar sus productos al consumidor final.

\section{Niveles de canal industrial o de bienes industriales}

Fabricantes - clientes industriales: Es el canal más común para los productos industriales, pues es el más corto y rápido.

Fabricantes - distribuidores industriales consumidores industriales: En este canal los distribuidores industriales ejecutan y desempeñan en algunas ocasiones las funciones de la fuerza de ventas del productor.

Fabricantes - representantes del fabricante distribuidores industriales - clientes industriales: Este tipo de canal, la función es facilitar las ventas mediante el agente, en tanto para el distribuidor que representa la tarea de almacenar los productos.

\section{Niveles de canal de servicio}

Productor - consumidor: Los servicios son de carácter intangible, sin embargo, con frecuencia se tiene acercamiento con la persona que brinda el servicio.

Productor - agente - consumidor: Por lo general estos servicios se dan de manera directa, sin embargo, existen excepciones, en donde un agente es el intermediario entre el fabricante y comprador y es precisamente este el que se encarga de efectuar las transacciones entre ellos. 


\section{Producción y ventas del limón en Ecuador}

Según el Ministerio de Agricultura y Desarrollo Rural de Colombia (2005) existen las siguientes cuatro variedades de limón: Citrus Limón Eureka, Citrus Lime Latifolia, Citrus Lime Aurantifolia, Citrus Lime Limetta. En Ecuador se cultiva básicamente el limón Sutil (Citrus Lime Aurantifolia) que está destinado para el consumo local y el limón Tahití (Citrus Lime Latifolia) puesto que es una variedad muy demandada por el comercio internacional.

Los principales problemas que tiene la producción de limón a nivel mundial son los cambios climáticos, las bacterias cítricas, la falta de cumplimiento de la calidad agroalimentaria, poca estimulación de la demanda interna y la escasez de formación en ventas internacionales (Hernandez Garnica \& Olvera Hernandez, 2010).
De acuerdo con el análisis de la información publicada por el (Instituto Nacional de Estadísticas y Censos. Encuesta de Superficie y Producción Agropecuaria Continua, 2019) que consta en la Ilustración 1, la producción en Ecuador ha sufrido diversas variaciones al transcurrir el tiempo debido al ingreso de limón de contrabando especialmente de Perú puesto que, por las condiciones climáticas muy parecidas a las nuestras, existen unas 12000 hectáreas del mismo tipo de limón, pero con mejor tecnología que les permite mayores rendimientos y con ello abastecen de la fruta al consumo interno (Valarezo Cely, 2019).

De acuerdo con la información proporcionada por el Gobierno Autónomo Descentralizado del Cantón Santa Ana, las hectáreas plantadas de limón en el año 2016 en la parroquia Ayacucho fueron 176,55 ha. y la producción del limón estimada anual fue de 47.370 pacas.

Tabla 1

Producción total de limón en Ecuador. Período 2016 - 2018

\begin{tabular}{cccccc}
\hline \multirow{2}{*}{ AÑO } & \multicolumn{2}{c}{ SUPERFICIE (HA) } & $\begin{array}{c}\text { PRODUCCIÓ } \\
\text { N (TM) }\end{array}$ & $\begin{array}{c}\text { VENTAS } \\
(\mathrm{TM})\end{array}$ & $\begin{array}{c}\text { VARIACIÓN } \\
\%\end{array}$ \\
\cline { 2 - 3 } & PLANTADA & COSECHADA & & & \\
\hline 2016 & 6.308 & 4.673 & 28.881 & 27.945 & - \\
2017 & 8.152 & 5.487 & 30.702 & 29.315 & $6 \%$ \\
2018 & 5.726 & 4.619 & 24.144 & 23.143 & $-21 \%$ \\
\hline
\end{tabular}

Fuente: INEC 2019. Elaborado por autores. 
Tabla 2

Distribución geográfica de la producción en toneladas métricas (TM) de limón en Ecuador. Período 2016-2019

\begin{tabular}{|c|c|c|c|c|}
\hline & & \multicolumn{3}{|l|}{$A \tilde{N} O S$} \\
\hline & & 2016 & 2017 & 2018 \\
\hline \multirow{12}{*}{ 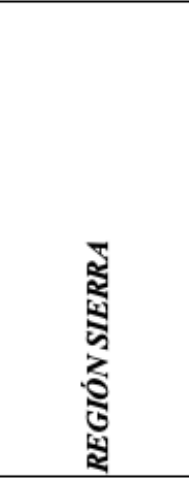 } & Azuay & 18 & 25 & 199 \\
\hline & Bolívar & 0 & 0 & 0 \\
\hline & Cañar & 0 & 2 & 2 \\
\hline & Carchi & 385 & 780 & 538 \\
\hline & Cotopaxi & 0 & 0 & 0 \\
\hline & Chimborazo & 14 & 0 & 55 \\
\hline & Imbabura & 1199 & 2093 & 1220 \\
\hline & Loja & 1018 & 807 & 533 \\
\hline & Pichincha & 1953 & 2178 & 2475 \\
\hline & Tungurahua & 43 & 21 & 0 \\
\hline & Santo Domingo de los Tsáchilas & 4 & 11 & 158 \\
\hline & \begin{tabular}{|l|} 
Total Región Sierra \\
\end{tabular} & 4634 & 5917 & 5180 \\
\hline \multirow{7}{*}{ 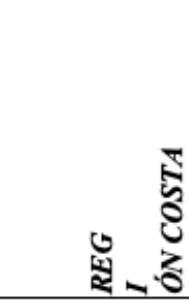 } & El Oro & 6736 & 11976 & 4641 \\
\hline & Esmeraldas & 25 & 322 & 366 \\
\hline & \begin{tabular}{|l|} 
Guayas \\
\end{tabular} & 671 & 61 & 1087 \\
\hline & Los Ríos & 58 & 36 & 0 \\
\hline & Manabí & 16015 & 11534 & 12074 \\
\hline & Santa Elena & 644 & 655 & 666 \\
\hline & Total Región Costa & 24149 & 24584 & 18834 \\
\hline \multirow{7}{*}{ 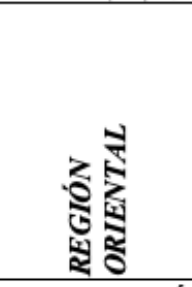 } & Morona Santiago & 98 & 0 & 0 \\
\hline & Napo & 0 & 0 & 80 \\
\hline & Orellana & 0 & 0 & 0 \\
\hline & \begin{tabular}{|l|} 
Pastaza \\
\end{tabular} & 0 & 201 & 50 \\
\hline & Sucumbíos & 0 & 0 & 0 \\
\hline & Zamora Chinchipe & 0 & 0 & 0 \\
\hline & Total Región Oriental & 98 & 201 & 130 \\
\hline \multicolumn{2}{|c|}{ PRODUCCIÓN NACIONAL TOTAL } & 57664 & 61203 & 48158 \\
\hline
\end{tabular}

Fuente: INEC 2019. Elaborado por autores

\section{MATERIALES Y MÉTODOS}

El tipo de investigación utilizado en el presente trabajo fue bibliográfico que es la primera etapa del proceso investigativo que proporciona el conocimiento de las investigaciones ya existentes que permitió desarrollar el estado del arte, según Arias (2012) es un proceso basado en la búsqueda, recuperación, análisis, crítica e interpretación de datos secundarios, es decir, los obtenidos y registrados por otros investigadores en fuentes documentales: impresas, audiovisuales o electrónicas, el análisis documental es una forma de investigación técnica, un conjunto de operaciones intelectuales, que buscan describir y representar los documentos de forma unificada sistemática para facilitar su recuperación.

Además, se empleó la investigación de campo que según el autor Arias (2012) es aquella que 
consiste en la recolección de datos directamente de los sujetos investigados o de la realidad donde ocurren los hechos (datos primarios), sin manipular o controlar variable alguna, es decir, el investigador obtiene la información pero no altera las condiciones existentes, permitiendo extraer datos e informaciones a través del uso de técnicas de recolección como entrevista y encuesta.

Los principales métodos empleados en la investigación fueron: método deductivo utilizado para analizar diversas informaciones que permitieron identificar los productores de limón que fortalecen a la economía de la parroquia y, los tipos de canales de distribución que utilizan actualmente. El método inductivo permitiendo obtener información referente al entorno actual de los productores de limón de la parroquia Ayacucho con la finalidad de conocer la forma o manera de distribución del producto hacia los diferentes mercados.
La población que se consideró dentro de este proyecto de investigación fueron productores de limón de la parroquia Ayacucho, entrevistándose a 49 de ellos y una entrevista a la Presidenta del GAD Parroquial de Ayacucho Lcda. Janeth Cevallos García, según datos proporcionados por el GAD del Cantón Santa Ana.

\section{RESULTADOS Y DISCUSIÓN}

La importancia del sector agropecuario en la economía nacional ha quedado evidenciada a lo largo de la historia económica y social de Ecuador, puesto que genera empleo, la balanza comercial del sector es altamente favorable y su aporte al PIB es relevante, además atrae la inversión extranjera directa con el firme propósito de reducir los índices de pobreza rural, inseguridad alimentaria y de bajos ingresos familiares, especialmente de los pequeños productores.

Figura 1

Edad de los productores de limón.

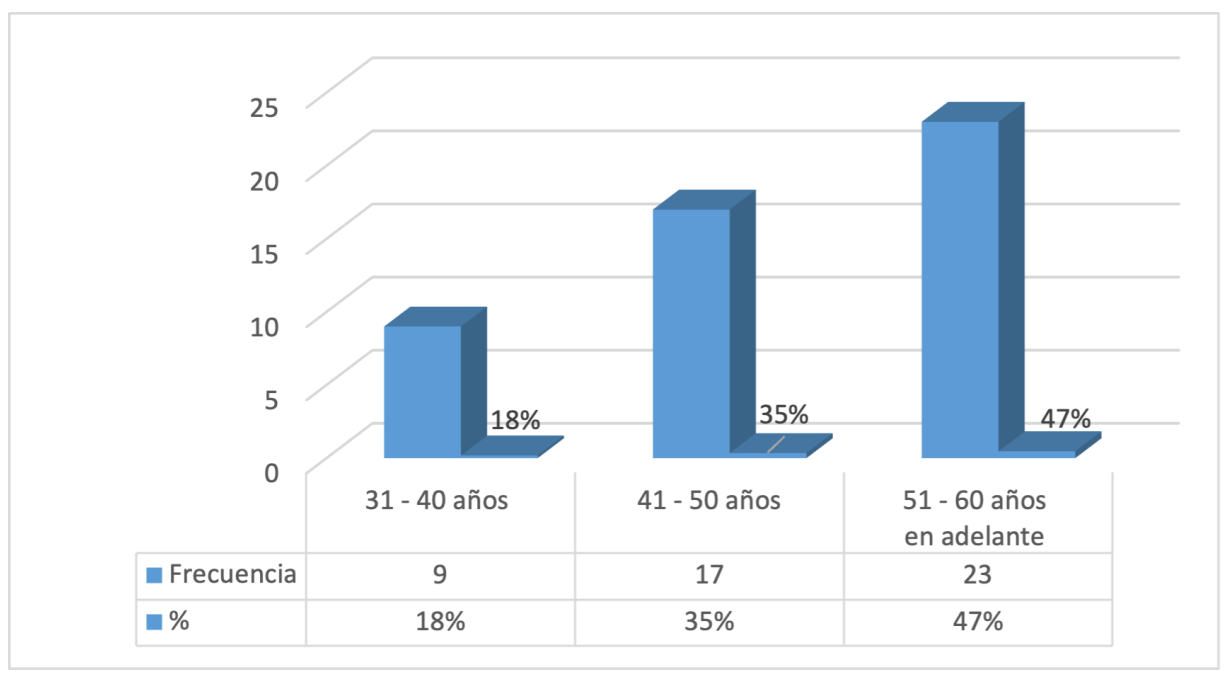

Fuente: Productores de limón de la Parroquia Ayacucho. Elaborado por: Autores. 
R E V V I

Publi c a n d o

I S S N $13390 \begin{array}{rlllll}9 & 3 & 0 & 4\end{array}$

Figura 2

Género de los productores de limón.

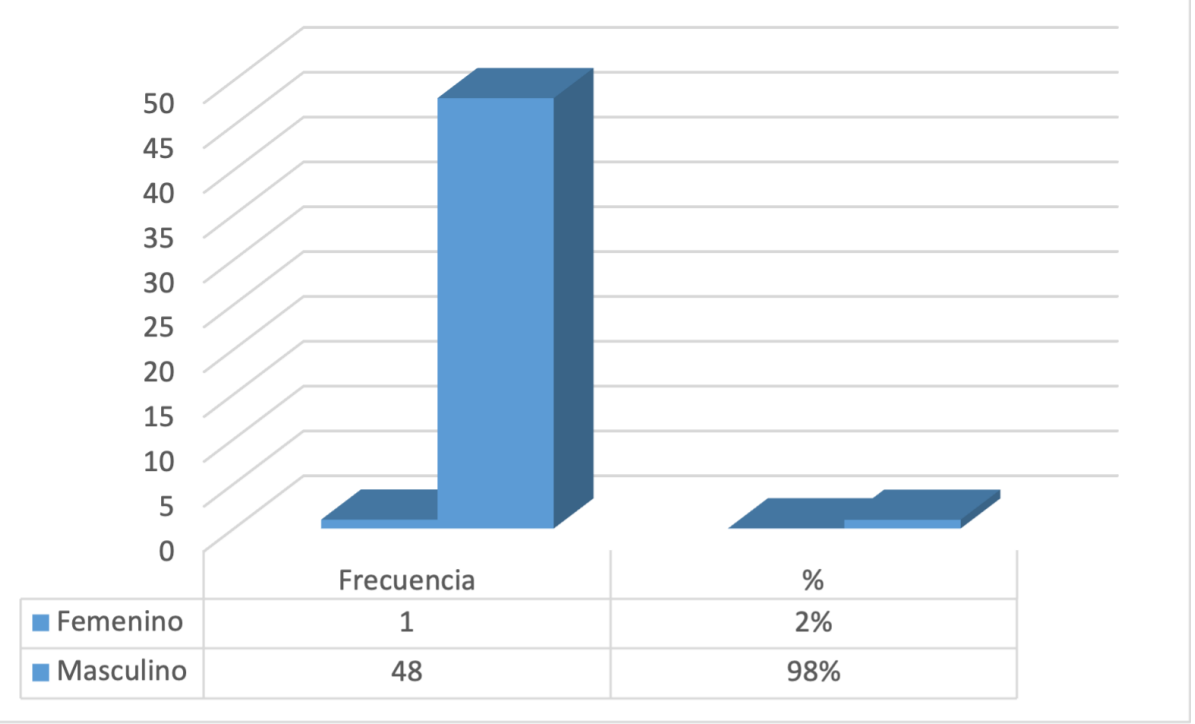

Fuente: Productores de limón de la Parroquia Ayacucho. Elaborado por: Autores.

\section{Figura 3}

Nivel de educación de los productores de limón.

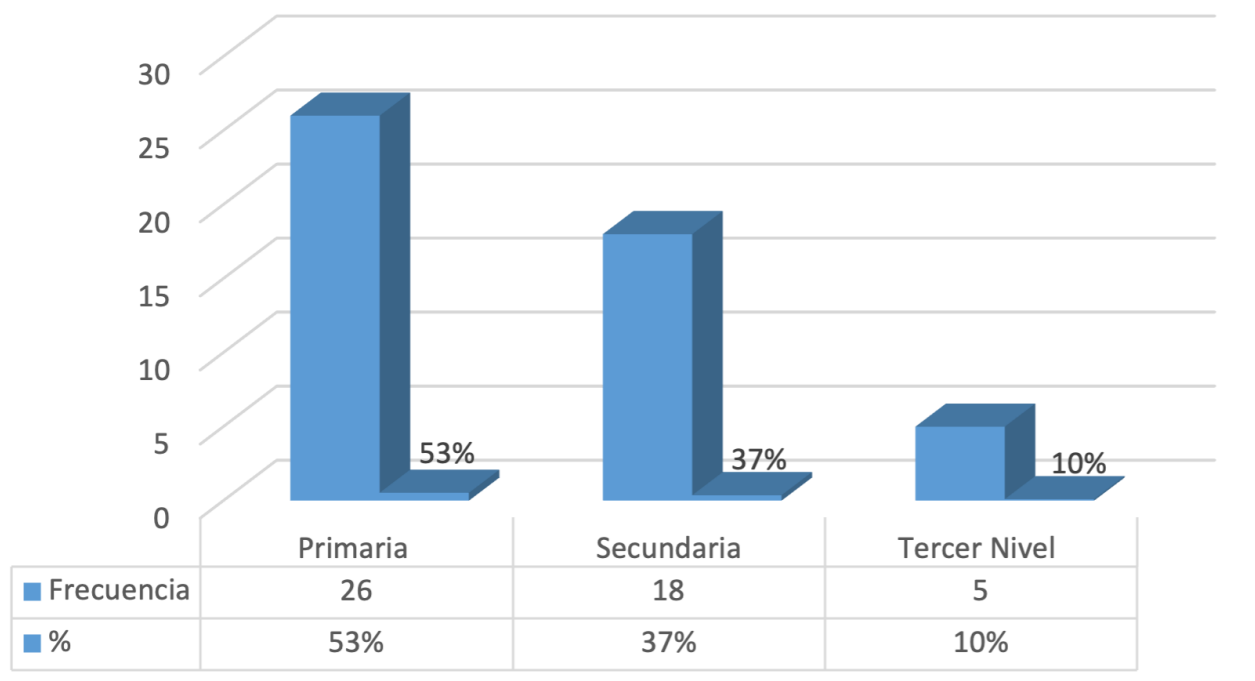

Fuente: Productores de limón de la Parroquia Ayacucho. Elaborado por: Autores. 


\section{Figura 4}

Tipo de canal de distribución utilizado para la distribución de su producto.

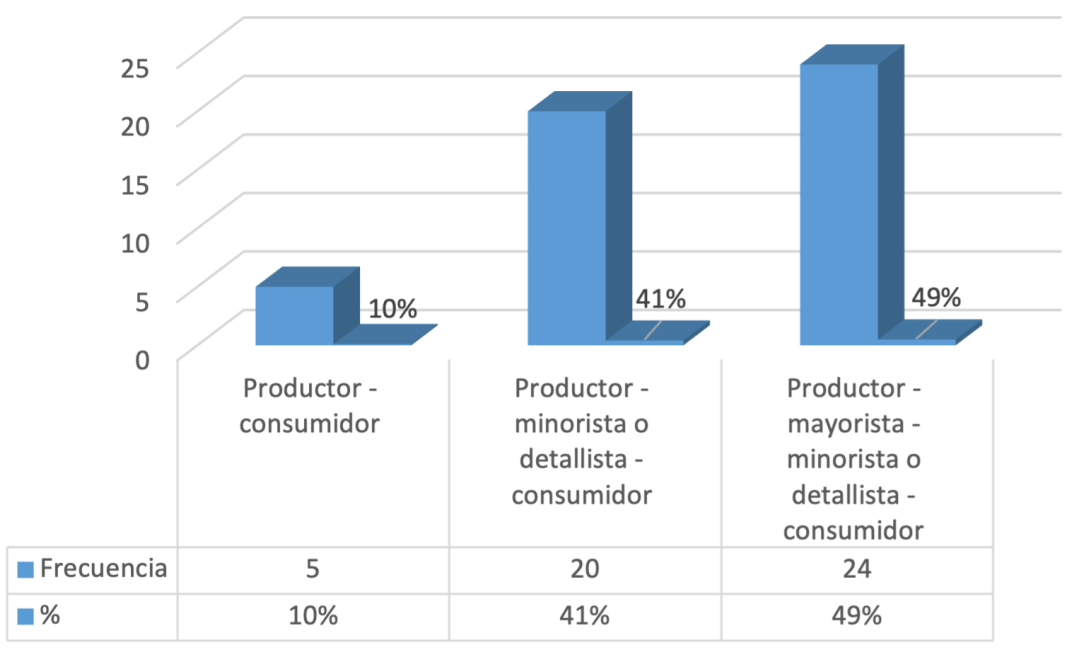

Fuente: Productores de limón de la Parroquia Ayacucho. Elaborado por: Autores.

\section{Figura 5}

Tipo de canal de distribución que considera se debe implementar para incrementar sus ventas.

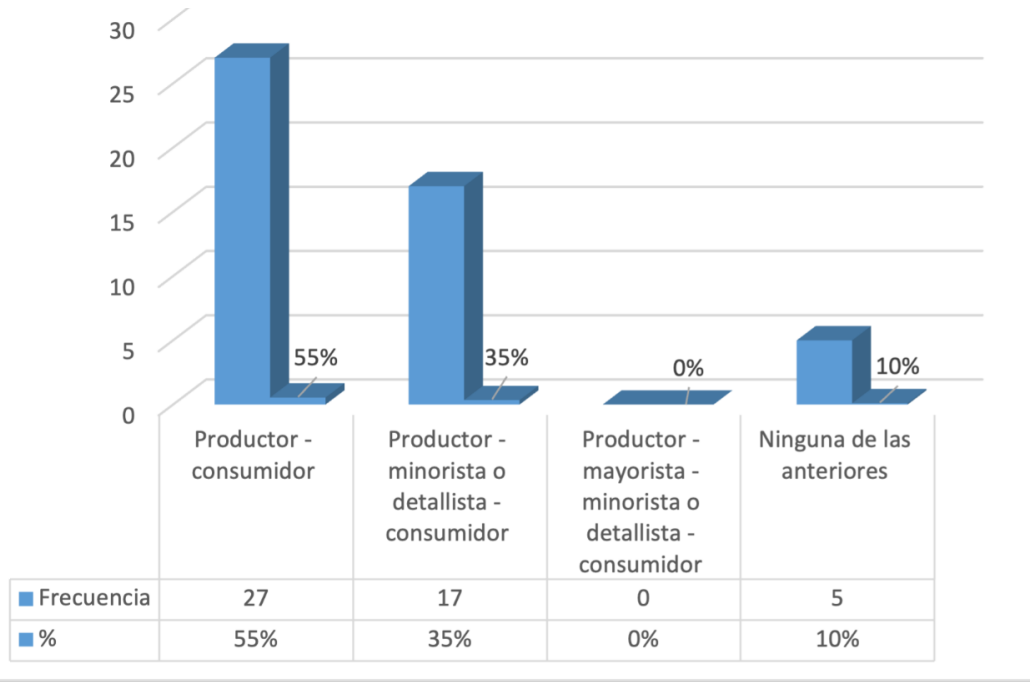

Fuente: Productores de limón de la Parroquia Ayacucho. Elaborado por: Autores. 


\section{Figura 6}

¿De qué manera la producción de limón beneficia a los habitantes de la parroquia?

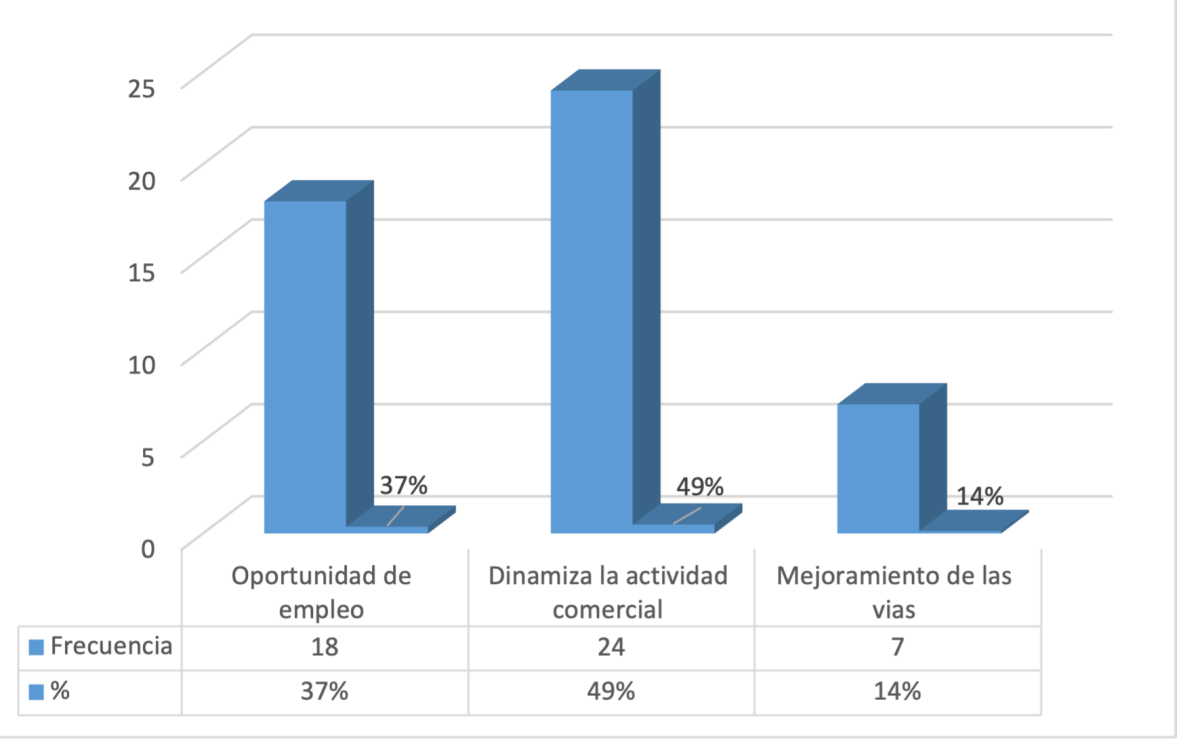

Fuente: Productores de limón de la Parroquia Ayacucho. Elaborado por: Autores.

\section{Figura 7}

Número de personas que se emplea por año en la producción de limón.

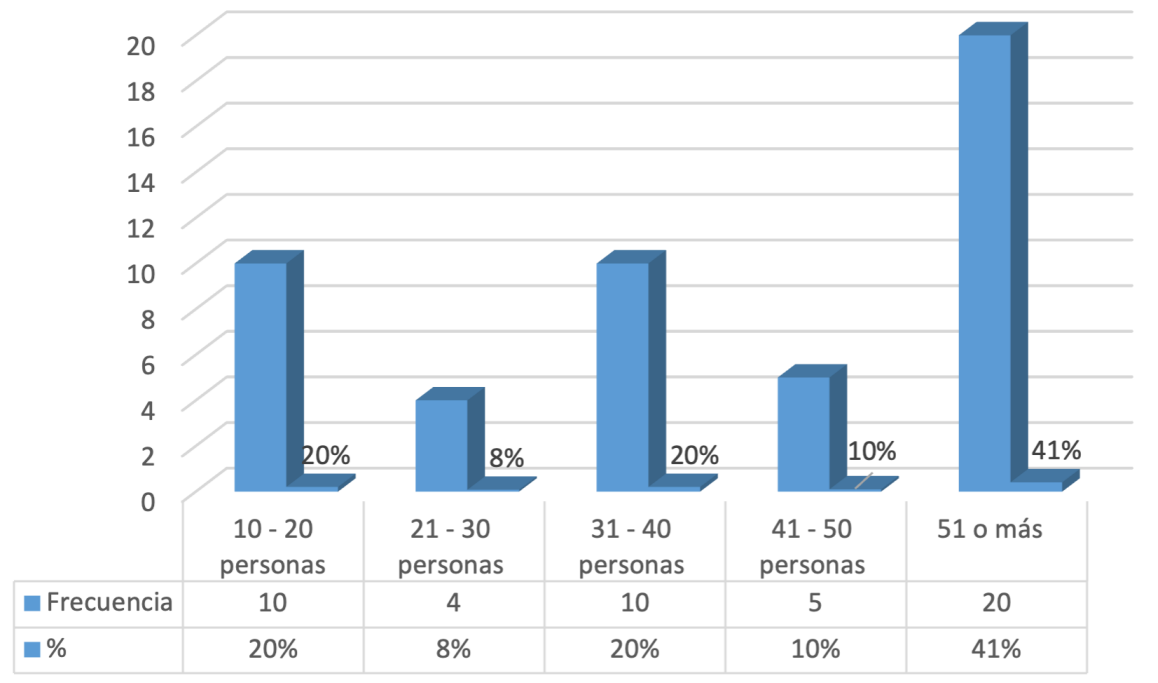

Fuente: Productores de limón de la Parroquia Ayacucho. Elaborado por: Autores. 
Figura 8

Tipo de canal de distribución que utiliza para la distribución de su producto.

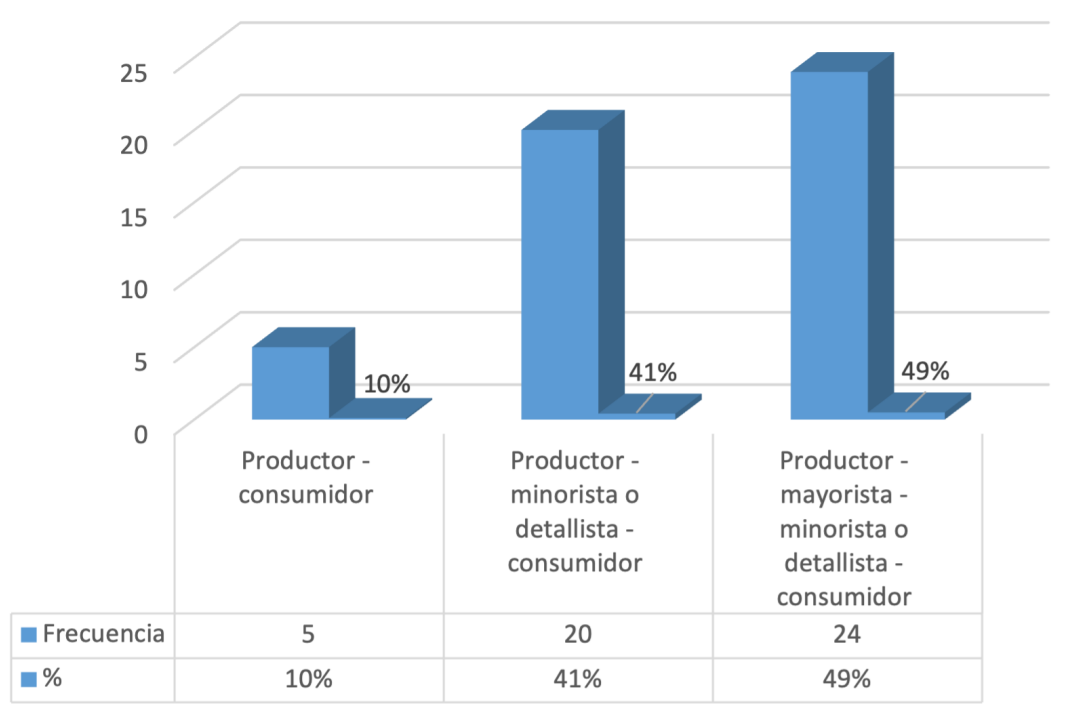

Fuente: Productores de limón de la Parroquia Ayacucho. Elaborado por: Autores.

\section{Figura 9}

¿Cree usted que nuevos canales de distribución mejorarían sus niveles de ventas?

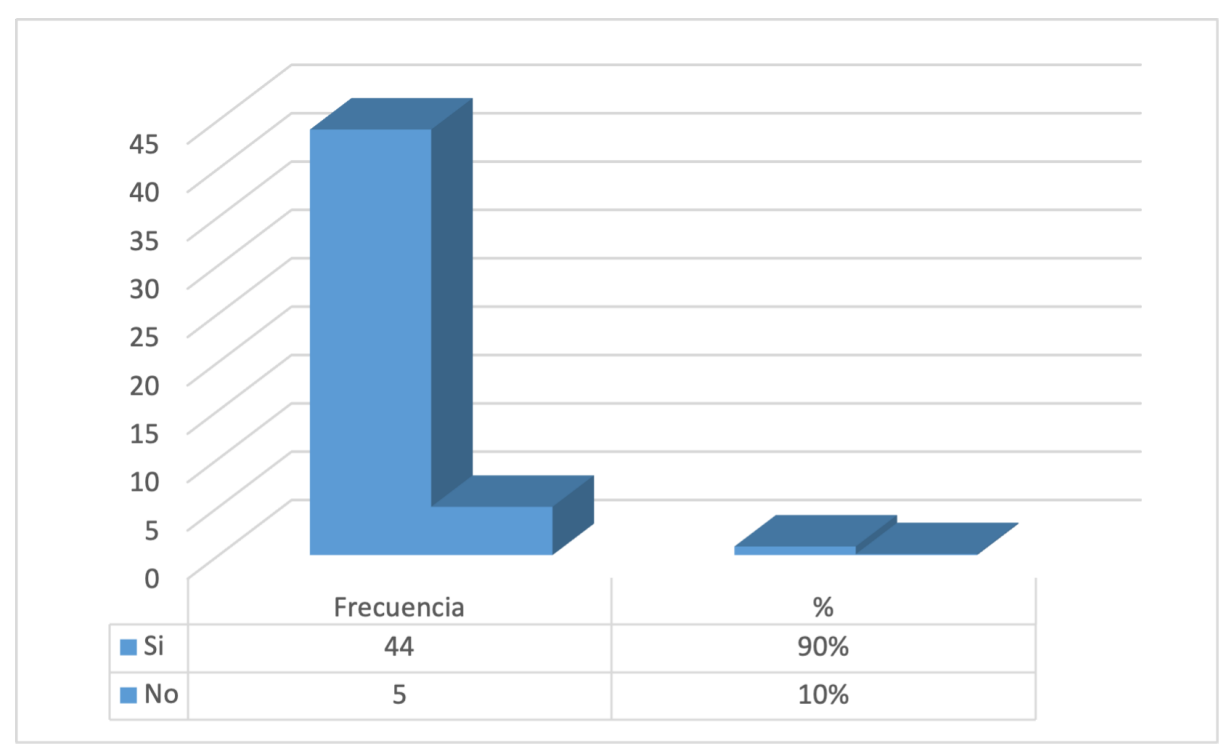

Fuente: Productores de limón de la Parroquia Ayacucho. Elaborado por: Autores. 
De acuerdo con los resultados de la investigación se pudo establecer que los productores de limón que fortalecen la economía de la parroquia Ayacucho son los que generan oportunidad de empleo, dinamizan la actividad comercial y ayudan al mejoramiento de las vías por la gestión que realizan los productores para que el GAD Cantonal les ayude en el mejoramiento de la carretera con la finalidad de trasladar su producto hacia el mercado. Mediante la encuesta realizada se evidenció que la población considera que la producción de limón los beneficia en $37 \%$ con oportunidades de empleo, el $49 \%$ porque dinamiza la actividad comercial, situación que fue corroborada por la presidenta de la Junta Parroquial de Ayacucho quien indicó que la producción de limón genera fuente de trabajo y de ingresos económicos.

Un canal de distribución es un conjunto de instituciones interdependientes que ayudan a que un producto o servicio se encuentre disponible para su uso o consumo por el comprador o el usuario corporativo. Entre los principales canales de distribución que utilizan actualmente los productores de limón de la parroquia Ayacucho con mayor frecuencia se encuentra el canal de productor - mayorista - minorista o detallista consumidor, esto se debe a: la falta transporte para trasladar su producto hacia otros mercados locales o internacionales, poca asosiatividad entre los productores y porque no existen convenios con los supermercados que permitan comercializar el producto de manera directa al consumidor.

El 90\% de los productores de limón requiere de un nuevo canal de distribución para la obtención de mejores ingresos y utilidad en sus ventas puesto que esto les permitirá generar más ganancias y solventar los gastos de producción y comercialización de su producto.

Entre los canales de distribución que influyen en el incremento de las ventas de limón en la parroquia Ayacucho, se pudo evidenciar que desean implementar el canal de distribución de productor - consumidor, puesto que consideran que este canal les permitirá generar utilidad en su finca obteniendo mayores ventas en vista de que entregaría el producto directamente al consumidor final, otros productores indican que ellos requieren implementar el canal productor minorista o detallista - consumidor puesto que ellos no cuentan con transporte para trasladar su producto y por lo tanto necesitarían un minorista o detallista que les ayude en el envío de productos y una menor parte de productores manifiestan que no requieren ningún nuevo canal de distribución debido a que ellos ya cuentan con un canal que les genera ganancias.

El sector agrícola tiene una importancia vital para todos los países no importa que sean subdesarrollados o desarrollados. Ayuda a compensar la necesidad de alimentación de los ciudadanos. El sector agrícola en desarrollo continuará aumentando el nivel de prosperidad de los ciudadanos del país, fomentando empleo y contribuyendo al crecimiento de la economía del país. (Karamelikli, 2016). La importancia del sector agropecuario en la economía nacional ha quedado evidenciada a lo largo de la historia económica y social de Ecuador. Actualmente cubre el $95 \%$ de la demanda interna de los alimentos que consume la población; genera empleo al $25 \%$ de la población económicamente activa (PEA), después del petróleo es el más importante generador de divisas, la balanza 
comercial del sector es altamente favorable y su aporte en el PIB es relevante debido a que en los últimos años aporto con $8,4 \%$ del PIB total, a su vez fortalece el sistema financiero público, atrayendo la inversión extranjera directa con el firme propósito de reducir los índices de pobreza rural, inseguridad alimentaria y de bajos ingresos familiares, especialmente de los pequeños productores agrícolas (Pino Peralta et al., 2018).

De acuerdo con esta información y mediante la presente investigación se pudo identificar que los productores de limón fortalecen la economía de la parroquia Ayacucho mediante la generación de fuentes de empleo puesto que esto permite que los ingresos familiares mejoren facilitándole a las personas satisfacer sus necesidades básicas y además las beneficia porque dinamiza la economía de la parroquia favoreciendo la calidad de vida de los individuos.

Un canal de distribución consiste en el conjunto de personas y empresas comprendidas en la transferencia de derechos de un producto al paso de este del productor al consumidor o usuario de negocios final, el canal incluye siempre al productor y al cliente final del producto en su forma presente, así como a cualquier intermediario, como los detallistas y mayoristas. (Staton et al., 2007). Por otra se determina que "Un canal de marketing (también llamado canal de distribución) es una estructura de negocios de organizaciones interdependientes que participan en el proceso de tener disponible un producto o servicio para el uso de los consumidores finales" (Lamb et al., 2011).

Referente a las definiciones mencionadas anteriormente sobre el canal de distribución se puede determinar que el canal que utilizan actualmente los productores de limón de la parroquia Ayacucho es canal productor mayorista - minorista o detallista - consumidor por lo cual se puede evidenciar que los productores no están utilizando un canal adecuado en vista de que ellos no cuentan con transporte para trasladar su producto, poca asociatividad entre los productores y falta de convenios con supermercados lo que le impide hacer llegar el producto al consumidor directamente puesto que lo ideal para una distribución de un producto debe ser de productor al consumidor final.

Al analizar sobre los canales de distribución que deben implementar los productores de limón en la parroquia Ayacucho se pudo constatar que existen varios niveles para la distribución de bienes de consumo las cuales son: Fabricante consumidor que es también llamado canal directo, debido que no cuenta con niveles de intermediarios y los productores venden directamente a los compradores. Productores minoristas o detallistas - consumidores: Se designa como canal dos, debido a que tienen un nivel de intermediarios, a saber: los minoristas o detallistas. Productores - mayoristas - minoristas o detallistas - consumidores: Este tipo de canal tiene dos niveles de intermediarios y se denominan canal tres, puesto que se utiliza con reiteración en los pequeños comerciantes de alimentos y de medicinas o en aquellos productores que no tienen la capacidad de hacer llegar sus productos al consumidor final (Velazquez Velazquez, 2012).

Efectivamente de acuerdo con lo analizado se pudo evidenciar que los productores de limón de 
la parroquia Ayacucho deben implementar un nuevo canal de distribución que les permita incrementar las ventas que sería el canal de productor - consumidor puesto que no cuentan con niveles de intermediarios y los productores realizan la venta directamente al comprador, a su vez se pudo determinar que el canal productor minorista o detallista - consumidor también beneficiaría a los productores que no tienen conocimientos respectivos acerca de cómo comercializar su producto o la falta de liquidez que no le permite adquirir un vehículo para trasladar su producto hacia otros mercados.

\section{CONCLUSIONES}

Los productores de limón que cultivan en la Parroquia Ayacucho, son mayoritariamente hombres con edades entre $51-60$ años, con nivel de instrucción primaria, únicamente el $10 \%$ posee título de tercer nivel.

Los productores de limón que fortalecen la economía de la parroquia Ayacucho son los que generan fuentes de empleo y dinamizan la actividad comercial puesto que esto conlleva al mejoramiento de la calidad de vida de los habitantes de la parroquia y en especial de los pequeños productores que buscan su bienestar y obtener mejores ganancias y rentabilidad en sus fincas, a su vez permite que los trabajadores puedan mejorar sus ingresos y el de su familia satisfaciendo sus necesidades básicas.

El canal de distribución que utilizan actualmente los productores de limón de la parroquia Ayacucho para promover la distribución de sus productos es el canal productor - mayorista - minorista o detallista - consumidor este canal es empleado por los productores debido a que no cuentan con transporte que permita trasladar su producto, la escasez de conocimiento entre los productores para crear una asociación de productores de limón y la falta de convenios entre supermercados.

Los productores de limón de la parroquia Ayacucho pueden implementar dos tipos de canales de distribución que son el canal directo donde no se utiliza intermediarios y genera mayor rentabilidad o el canal dos donde se utiliza un minorista o detallista, pero se realiza por la falta de transporte que no le permite hacer llegar el producto de manera directa hacia otros mercados locales o nacionales con la desventaja de que el precio varía por el costo de transporte. 


\section{REFERENCIAS BIBLIOGRÁFICAS}

Arias, F. G. (2012). El proyecto de investigación: Introducción a la metodología científica (Sexta ed.). Caracas: Epistema.

Armstrong, G., \& Kotler, P. (2013). Fundamentos de Marketing. México: Pearson Educación.

Arreaga Gómez, L. (2017). La producción y exportación de las principales frutas no tradicionales y su importancia en las exportaciones totales de Ecuador, período 2012 - 2016. Guayaquil: Universidad de Guayaquil. Obtenido de http:// repositorio.ug.edu.ec/bitstream/redug/ 23040/1/TESIS\%20FINAL.pdf

Chirinos, C. (2011). Nicho de mercado: El enfoque desde el oceano azul . REDALYC.

Colombia IICA. (1996). Informe del Taller de Estadistica con Enfoque de Genero Subregion Andina. Colombia : IICA Biblioteca Venezuela.

De la Riva Del Valle, S. (2018). Recursos Biblio. Obtenido de http:// recursosbiblio.url.edu.gt/tesisjrcd/ 2018/06/14/DeLaRiva-Santiago.pdf

Diez de Castro, E., \& Navarro García, A. (1994). Distribución Comercial. España: Mc Graw Hill.

Dirección de Inteligencia Comercial e Inversiones. (2013). Pro- Ecuador. Análisis sectorial de frutas no tradicionales. Pro-Ecuador. Obtenido de https://www.proecuador.gob.ec/ w pcontent/uploads/2013/11/ PROEC_AS2012_FRUTAS.pdf
Ecuavisa. (14 de 09 de 2017). Ecuavisa. Obtenido de https://www.ecuavisa.com/articulo/ noticias/nacional/319616-agricultorespreocupados-baja-produccion-del-limon

El Comercio. (19 de 02 de 2011). El Comercio. Obtenido de https://www.elcomercio.com/ actualidad/negocios/cuatro-variedades-delimon-de-1.html

Española, D. d. (s.f.). https://dle.rae.es. Obtenido de https://dle.rae.es/srv/fetch?id=bXt7EYJ

Espejo Martínez, A., \& Ramos Soto, A. L. (2017). Investigación y Desarrollo Económico Administrativo. México: TECCIS A.C.

Figueroa, A. M. (2015). Situación actual de los citricultores del Valle del Río Valdivia, Santa elena; bajo el enfoque del marco de capitales. Santa Elena: Universidad Estatal Península de Santa Elena.

Fischer, L., \& Espejo, J. (2011). Mercadotecnia. México: McGraw Hill.

FRED R , D. (2003). Concepto de Administracción Estratégica. México: PEARSON EDUCACIÓN.

Freire Rubio, M. T., \& Blanco Jimenez , F. J. (2006). Prácticas y Conceptos Básicos de Microenomía. Madrid: ESIC.

GAD Parroquial Ayacucho. (2015 - 2019). Plan de Desarrollo Territorial Ayacucho.

Garcia Bobadilla, L. M. (2009). + Ventas. Madrid: ESIC.

Garcia Duque, B. K. (2014). UCSG. Obtenido de http://repositorio.ucsg.edu.ec/bitstream/ 
$\begin{array}{lllllll}\text { R } & \text { E } & \text { V } & \text { I } & \text { S } & \text { T } & \text { A }\end{array}$

$3317 / 2710 / 1 / T-U C S G-P R E-T E C-$ EADR-13.pdf

Geilfus, F. (1994). El Arbol al Servicio del Agricultor . Costa Rica: CATIE.

Hernández Garnica, C., \& Olvera Hernandez, J. C. (04 de 2010). ACACIA. Obtenido de http:// a cacia.org.mx / bu s queda/pdf/ IMPACTO_DE_LA_TECNOLOG_A_RE GIONAL.pdf

INFOCAMPO. (16 de 07 de 2017). INFOCAMPO. Obtenido de https:// www.infocampo.com.ar/argentina-es-elprimer-productor-mundial-de-limones/

Instituto Nacional de Estadísticas y Censos. Encuesta de Superficie y Producción Agropecuaria Continua. (2019). Ecuador en cifras. Obtenido de Información Estadísitica: https://www.ecuadorencifras.gob.ec/ encuesta-de-superficie-y-produccionagropecuaria-continua-bbd/

Jerez, M. T. (2016). Canales de distribución en el marketing MIX.

Jimenez, S. M. (2012). Distribución Comercial Aplicada. Madrid, España: ESIC.

Karamelikli, H. (09 de 03 de 2016). TRT Español. Obtenido de https://www.trt.net.tr/espanol/ programas/2016/03/09/por-que-es-tanimportante-el-sector-agricola-para-laeconomia-de-pais-447371

Kennedy, G. D. (2014). Repositorio UCSG. Obtenido de http://repositorio.ucsg.edu.ec/bitstream/ $3317 / 2710 / 1 /$ T - UCS G - PRE - TEC EADR-13.pdf

Kossen, S. (1992). La venta de creativa. Madrid:
Ediciones Diaz de Santos S\&A.

Lamb, C., Hair, J., \& McDaniel, C. (2011). Marketing. México: Cengage Learning.

León Alvéstegui, G., Valdéz Rodríguez, H., \& Vásquez Mamani, V. H. (2003). Mercado KANTUTA ¿Un mercado campesino? La Paz : Bolsillo.

Leon, J. (1987). Botánica de los Cultivos Tropicales . San Jose, Costa Rica : IICA.

Ley Organica Popular y Solidaria. (23 de 10 de 2018). SEPS. Obtenido de http://www.seps.gob.ec/ d o c u ments / $20181 / 25522 /$ LEY\%20ORGANICA\%20DE\%20ECONO MIA\%20POPULAR\%20Y\%20SOLIDARI A\%20actualizada\%20noviembre\%202018. p d f / 66 b 23 e e f - 8 b $87-4$ e 3 a boba-194c2017e69a

Little , E., Wadsworth, F., \& Marrero, J. (1977). Árboles comunes de Puerto Rico y las Islas Virgenes. Puerto Rico: Universidad de Puerto Rico.

Ministerio de Agricultura y Desarrollo Rural. (2005). La competitividad de las cadenas agroproductivas en Colombia 1991- 2004. Bogota: IICA.

Opportimes. (05 de 05 de 2020). Opportimes. Obtenido de Los diez mayores exportadores de limones del mundo: https:// www.opportimes.com/los-10-mayoresexportadores-de-limones/

Pérez Porto, J., \& Merino, M. (2012). Definición.de. Obtenido de https://definicion.de/ parroquia/

Perez, A. V. (2011). Técnica de venta. Antequera: 
Innovación y Cualificación.

Pino Mantilla, C., Soria Altamirano, C., \& Espinel Martínez, R. (2009). Estudio de prefactibilidad para la producción del limón tahiti de la Península de Santa Elena. Tesis de grado, Guayaquil. Obtenido de https:// www.dspace.espol.edu.ec/bistream/ 123456789/471/1/923.pdf

Pino Peralta, S., Apolo Loayza, A., Sisalema Morejon, L., \& Aguilar , H. R. (2018). Aporte del sector agropecuario a la economía de Ecuador. Revista ESPACIOS. Obtenido de https://www.revistaespacios.com/ a18v39n32/a18v39n32p07.pdf

Ruiz Gonzalez , A. E., \& Wang Quezada, L. A. (2015). Repositorio UCSG. Obtenido de http:// repositorio.ucsg.edu.ec/bitstream/ $3317 / 4370 / 1 /$ - UCS G - PRE - ESP CFI-176.pdf

Santistevan Mendez, M. S. (2016). Repositorio Molina. Obtenido de http:// repositorio.lamolina.edu.pe/bitstream/ handle/UNALM/2740/F01-S3558T.pdf? sequence $=1$ \&isAllowed $=\mathrm{y}$

Statista. (01 de 2021). Statista. Obtenido de Ranking de los mayores países productores de limones y limas a nivel mundial en 2019.: https://es.statista.com/estadisticas/613493/ principales-paises-productores-de-limonen-el-mundo/\#statisticContainer

Staton, W., Etzel, M., \& Walker , B. (2007). Fundamentos de Marketing 14th Edicion. Mexico: McGraw-Hill Interamericana.

Valarezo Cely, O. (19 de 04 de 2019). El Diario. Obtenido de http://www.eldiario.ec/ noticias-manabi-ecuador/500179agroindustria-del-limon/

Vásquez, L., \& Vásquez, P. (2014). Producción de limón Tahiti (Citrus Latifolia Tan.) con la aplicación de humus de lombriz. Revista científica agrociencias Amazonía, 21(1), $1-6$.

Velazquez Velazquez, E. (2012). Canales de Distribución y Logística. México: Red Tercer Milenio.

政

